\title{
Definitions of groove and hollowness of the infraorbital region and clinical treatment using soft-tissue filler
}

\author{
Ji-Hyun Lee ${ }^{1}$, Giwoong Hong ${ }^{2}$ \\ ${ }^{1}$ Department of Anatomy, Gachon University School of Medicine, Incheon; ${ }^{2}$ Samskin Plastic Surgery Clinic, Seoul, Korea
}

\begin{abstract}
Clarification is needed regarding the definitions and classification of groove and hollowness of the infraorbital region depending on the cause, anatomical characteristics, and appearance. Grooves in the infraorbital region can be classified as nasojugal grooves (or folds), tear trough deformities, and palpebromalar grooves; these can be differentiated based on anatomical characteristics. They are caused by the herniation of intraorbital fat, atrophy of the skin and subcutaneous fat, contraction of the orbital part of the orbicularis oculi muscle or squinting, and malar bone resorption. Safe and successful treatment requires an optimal choice of filler and treatment method. The choice between a cannula and needle depends on various factors; a needle is better for injections into a subdermal area in a relatively safe plane, while a cannula is recommended for avoiding vascular compromise when injecting filler into a deep fat layer and releasing fibrotic ligamentous structures. The injection of a softtissue filler into the subcutaneous fat tissue is recommended for treating mild indentations around the orbital rim and nasojugal region. Reducing the tethering effect of ligamentous structures by undermining using a cannula prior to the filler injection is recommended for treating relatively deep and fine indentations. The treatment of mild prolapse of the intraorbital septal fat or broad flattening of the infraorbital region can be improved by restoring the volume deficiency using a relatively firm filler.
\end{abstract}

\section{Keywords Tear trough / Skin aging / Eyelids / Subcutaneous tissue / Dermal fillers}

Received: 14 Jul 2017 • Revised: 17 Jan 2018 • Accepted: 25 Jan 2018

pISSN: 2234-6163 • elSSN: 2234-6171 • https://doi.org/10.5999/aps.2017.01193 • Arch Plast Surg 2018;45:214-221
Correspondence: Giwoong Hong Samskin Plastic Surgery Clinic, 6 Nonhyeon-ro 175-gil, Gangnam-gu, Seoul 06030, Korea

Tel: +82-2-3447-7755

Fax: +82-70-7008-7000

E-mail: cosmetic21@hanmail.net

\section{INTRODUCTION}

A groove in the infraorbital region (GIR) refers to a narrow furrow around the infraorbital rim, and hollowness of the infraorbital region (HIR) refers to a sunken space in the infraorbital area. Treatments for the correction of GIR and HIR are usually divided into nonsurgical and surgical corrections [1,2]. Among the nonsurgical correction options, filler injections are becom- ing more common than fat injections, because they are convenient and can be done repeatedly, if necessary, without additional fat harvesting [3]. However, the type of filler and injection method should be carefully selected according to the cause and severity of the groove or hollowness to ensure safe and optimal treatment. This goal can be achieved by clearly understanding the definitions and classification of GIR and HIR.

GIR has several names, including "nasojugal groove (fold)" 
and "tear trough deformity." The nasojugal fold was first described by Whitnall in 1932 [4], and the term "nasojugal groove" was first used by Loeb in 1993 [5]. The term "tear trough deformity" was first introduced by Flowers in 1970 $[6,7]$. Ever since, several terms that refer to GIR and HIR have been inconsistently used, confused, overlapped, and mistaken for one another, so Hwang attempted to refine these terms [7]. The terms "nasojugal groove" (or fold) and "tear trough deformity" were considered to refer the same concept until quite recently [7-10]. However, the 2 terms can be differentiated via anatomical studies, and it is possible to distinguish a nasojugal groove from a tear trough deformity clinically according to practical variations and the characteristics of the grooves and hollowness of patients' faces.

The goal of this article was to review the definitions and classification of GIR and HIR according to cause and appearance. We would like to promote safe and successful results by proposing optimally effective filler treatment methods based on the definitions and classification presented herein.

\section{DEFINITIONS AND ANATOMIC STUDIES OF GIRAND HIR}

GIR refers to a fine indentation located around the orbital rim at the lid-cheek junction, and has several names, including nasojugal groove (or fold), tear trough deformity, and palpebromalar groove [1,2,4-11]. In contrast, HIR refers to a relatively broad depression in the infraorbital area, including the medial and central cheek region, and an area of the orbital rim. It can occur with or without GIR [1].

Whitnall [4] first used the term "naso-jugal" in 1932, and he described the "nasojugal fold" as corresponding to a gap between the orbicularis oculi muscle (OOM) and the levator labii superioris alaeque nasi muscle (LLSAN). In 1993, Loeb [5] used the term "nasojugal groove."

"Tear trough deformity" was first used by Flowers in 1970, referring to "a deep diagonal groove at the junction of the eyelid and cheek" [6,7], and he described it as a groove caused by herniated or protruding orbital fat in $1991[7,12]$. The tear trough deformity is considered to be located $2-3 \mathrm{~mm}$ inferior to the orbital rim at the lid-cheek junction; it is present at the junction between the palpebral and orbital parts of the OOM, and this junction also corresponds precisely to the superior border of the malar fat pad [2].

Some authors have proposed that the groove at the lateral lidcheek junction based on the mid-pupillary line should be called the "palpebromalar groove" and that the tear trough deformity should be defined as the groove that exists medial to the mid- pupillary line $[13,14]$. Yang et al. [8] reported that the orbicularis retaining ligament (ORL) of the lateral orbital rim plays a significant role in the formation of a groove in the lateral portion of the lid-cheek junction. According to their study, the ORL arises from the lateral orbital thickening and ends lateral to the mid-pupillary line at the junction between the palpebral and orbital part of the OOM, and the ORL limits the descent of the OOM throughout aging. They defined the tear trough deformity as the indentation of the medial third of the orbital rim from the medial canthal area to the mid-pupillary line, and stated that it can be seen from a young age due to its anatomical characteristics. With aging, the palpebromalar groove of the middle and lateral orbit becomes visible, and it is usually looser than the medial indentation due to anatomical differences [9].

Many anatomical theories have been proposed regarding the causes of GIR and HIR [1,2,4-10]. The factors mentioned in these theories are (1) herniation of the intraorbital fat, (2) attachment of the orbital septum to the inferior orbital rim, (3) the prominence of the orbital rim, (4) atrophy of the skin and subcutaneous fat, including the bone in the suborbital area, (5) the presence of the ORL and tear trough ligament (TTL), (6) the gap between the OOM and LLSAN muscles, and (7) surface puckering caused by contraction of the OOM. Herniation of intraorbital fat is doubtless an important factor contributing to GIR. However, other proposals regarding related factors remain controversial.

In 2009, Haddock et al. [2] reported that the tear trough deformity is irrelevant to the arcus marginalis, the orbital rim, and the junction between the OOM and LLSAN muscle. He explained that atrophy of the skin and subcutaneous tissue is the most plausible explanation for the aging-related deepening of GIR. In 2013, Yang et al. [8] reported that atrophy of the malar fat pad makes GIR and HIR more obvious with age. He found that the superior border of the malar fat pad in elderly specimens was lower than in younger specimens, and the thickness and volume of those malar fat pads were visibly less than in young specimens. He speculated that if the malar fat pad in young people were thin, the shape of the OOM would be more visible, which is why a significant tear trough is visible in some younger individuals.

In 2012, Wong et al. [10] reported that the TTL is a true osteocutaneous ligament that exists in the medial suborbital region of the maxilla. He described the TTL as continuous with the ORL, which consists of the upper and lower lamella laterally, and stated that these 2 lamellae are associated with lateral orbital thickening. He also considered the TTL to be the main structure involved in the formation of the tear trough deformity. According to their study, TTL and ORL have the effect of tether- 
ing and holding the OOM and malar fat pad onto the skeleton. Among the factors that contribute to make the tear trough deformity distinct, the primary etiologic factor is the tethering effect of the TTL, which binds the medial suborbital skin to the maxilla.

According to the study by Wong et al. [10], the TTL exists in the medial suborbital region between the palpebral parts and the orbital parts from the level of the insertion of the medial canthal tendon to the mid-pupillary line. However, Haddock et al. [2] emphasized that there is no true ligament medially; instead, the OOM simply is firmly attached to the bone along the tear trough. Yang et al. [8] had a similar opinion and suggested that the TTL was an extension of the ORL. Moreover, Wong et al. [10] believed the TTL to be connected directly to the skin, but according to Haddock et al. [2], the ORL attaches the muscle to the skin along the lid-cheek junction. Yang et al. also found that the ORL was not connected directly to the skin, but ended at the malar fat pad after penetrating the OOM.

However, Hwang et al. [9] performed a parasagittal and horizontal section of the lower eyelid on cadavers and conducted a histological study in 2015; they could not find a TTL or ORL. They stated that they could not find a true ligament between the palpebral and orbital parts of the OOM in gross sections, and that there was no true ligament in the histological study. Instead, they observed many areas of fibrotic connective tissue between the muscle bundles of the OOM. They emphasized the OOM attached to the periosteum along the tear trough, and then stated that "while squinting, the OOM, the protractor of the eyelid, contracts and the vertical width shortens, and thereafter, a surface puckering of the delicate periorbital skin appears along with the origin of the OOM." Therefore, the TTL and ORL remain controversial, and it seems that further study should be done to investigate these structures.

Although Haddock et al. [2] described GIR as being unrelated to the junction between the LLSAN and the OOM, in 2012, Stutman and Codner [14] arrived at a different opinion. In their review article, they pictured and described the "incorrect tear trough" as located between the orbital portion of the OOM and LLSAN, and the "correct tear trough" as located between the palpebral and orbital part of OOM. Similarly, Kim et al. [15] explained the tear trough deformity and nasojugal groove separately in their textbook, and described the location of nasojugal groove as corresponding to that of the inferior border of the OOM. While many previous studies have described the tear trough deformity and nasojugal groove as the same structure, they have different features based on clinical and anatomical findings (Fig. 1).

Originally, Whitnall [4] described the "naso-jugal" fold as oc-

\section{Fig. 1. Difference of tear trough and nasojugal groove}

Tear trough deformities are located between the palpebral and orbital parts of the orbicularis oculi, and the location of the nasojugal groove corresponds to the inferior border of the orbicularis oculi. Blue dashed line, tear trough deformity; red dashed line, nasojugal groove; OF, orbital fat; OS, orbital septum; SOOF, sub orbicularis Oculi fat; PO, palpebral part of the orbicularis oculi muscle; LLSAN, levator labii superioris alaeque nasi muscle; MFP, malar fat pad; 00M, orbicularis oculi muscle.

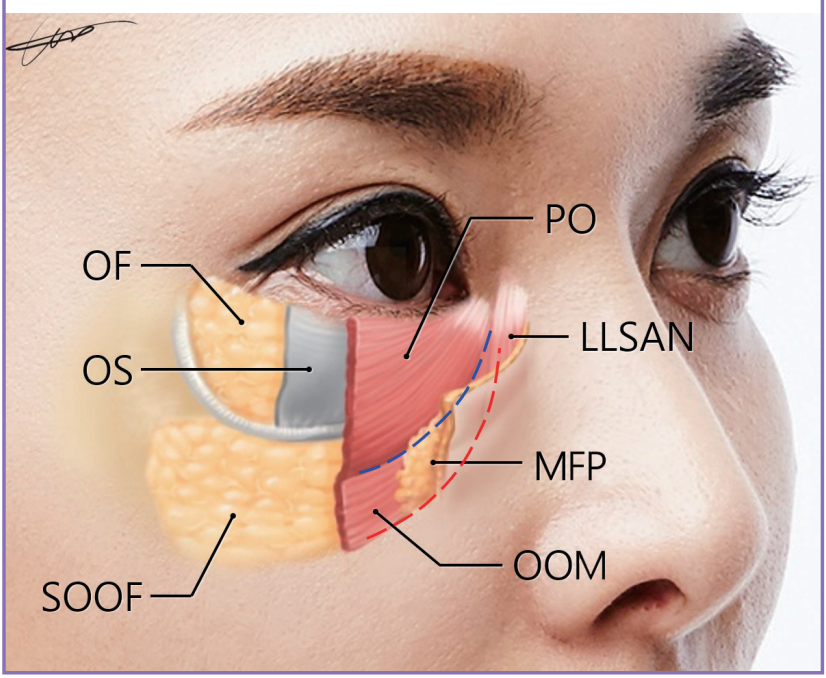

curring between the OOM and the head of LLSAN, as mentioned above, and the stated that the facial vein and artery run along its line. In addition, Flowers described the tear trough deformity as being caused by herniated or protruding orbital fat $[6,7]$; these explanations differ. According to their anatomical study of the facial artery, Yang et al. [16] found that the nasojugal portion of the detoured branch of the facial artery traveled along the inferior border of the OOM in approximately $30 \%$ of the Korean population and then ascended towards the forehead. In fact, this branch is the same as the infraorbital trunk of the duplex facial artery identified in a previous study by Koh et al. [17]. This result coincides precisely with Whitnall's description and indicates how the nasojugal groove corresponds to the position of the inferior border of the OOM. When describing the tear trough deformity, Flowers considered that the herniation of orbital fat would be limited in some extent by the orbital septum at the orbital rim and it would descend sufficiently to the inferior border of the OOM. Eventually, the terms were used for different structures; thus, we can suggest that tear trough deformity and nasojugal groove should be considered as meaningfully separate structures. This distinction can change how GIR and HIR are treated, as accounting for the different definitions and causes of the groove will help obtain safe and effective results. For instance, avoiding injury to the infraorbital or detoured branch of the facial artery is very important when treating a nasojugal 


\section{Fig. 2. The grooves of the infraorbital region}

This middle-aged female patient had a significant tear trough deformity and distinguishable nasojugal groove. PMG, palpebromalar groove; MCG, mid-cheek groove; $\Pi$ D, tear trough deformity; NG, nasojugal groove.

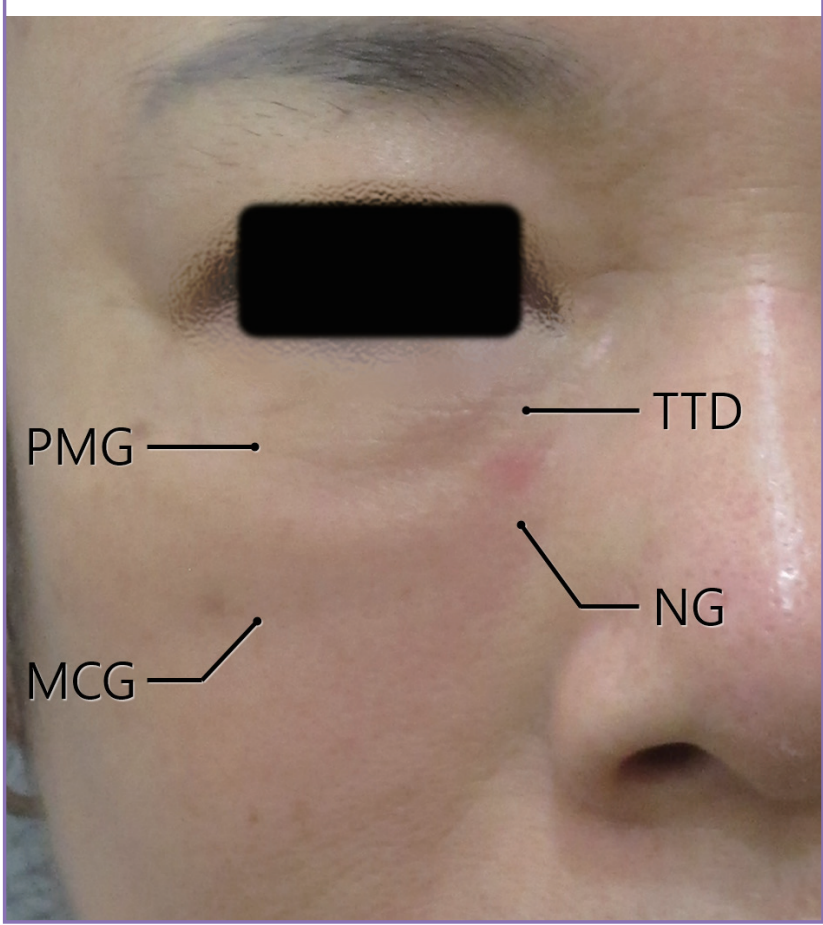

groove. In fact, there has been a slight oversight; in 2007, Bosniak et al. [18] already mentioned that the tear trough and nasojugal groove should be distinguished, and they proposed that "the tear trough be defined as only the superior aspect of the nasojugal groove that occurs in patients from all age groups." Actually, it has been shown that the nasojugal groove is located slightly inferior to the tear trough deformity and more obliquely. In some cases, the nasojugal groove can be seen as the medial part of the mid-cheek groove, and it seems to continue without separation, but the break point can be found with mild unevenness when viewed with care. In addition, it can be observed that there are different aspects of the 2 grooves in some patients who have both grooves in their infraorbital region (Fig. 2). The proposal of Bosniak et al. helps explain these cases with ease.

According to the above-described studies, GIR can be subdivided into the nasojugal groove and tear trough deformity, and we propose that the combined resultant causes of GIR and HIR are as follows. (1) When atrophy of skin and subcutaneous fat occurs in the suborbital area, the shape of the OOM may become prominent, appearing as a tear trough deformity or nasojugal groove (Fig. 3A-C). In such cases, the indentation is not severe and usually shows a mild depression without the presence of a dense ligamentous structure, but when there is a

\section{Fig. 3. Causes of groove or hollowness}

(A) Normal condition. (B) Appearance of tear trough deformity (TTD) through the atrophy of skin and subcutaneous fat in the suborbital area. (C) Appearance of nasojugal groove in the skin and subcutaneous fat in the suborbital area. (D) Appearance of TTD due to contraction of the orbital part of the orbicularis oculi muscle or squinting. (E) Appearance of TTD due to herniation of intraorbital fat. (F) Appearance of infraorbital hollowness through malar bone resorption with soft tissue atrophy.

(A)

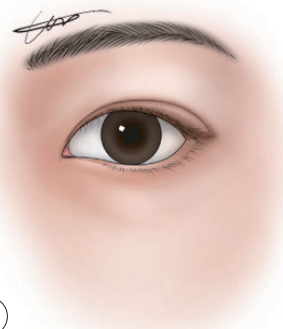

(D)

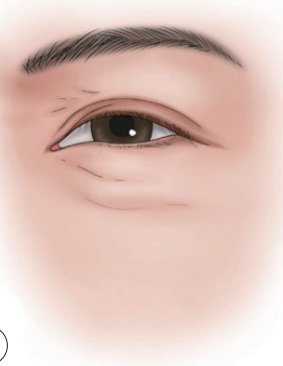

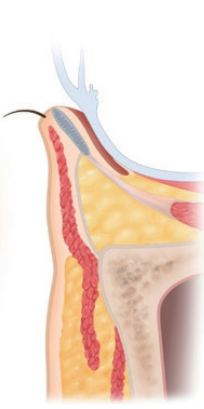

B
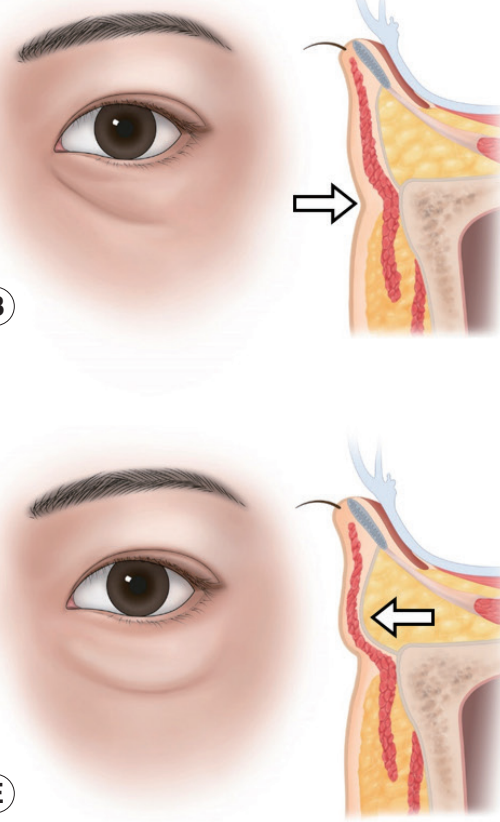

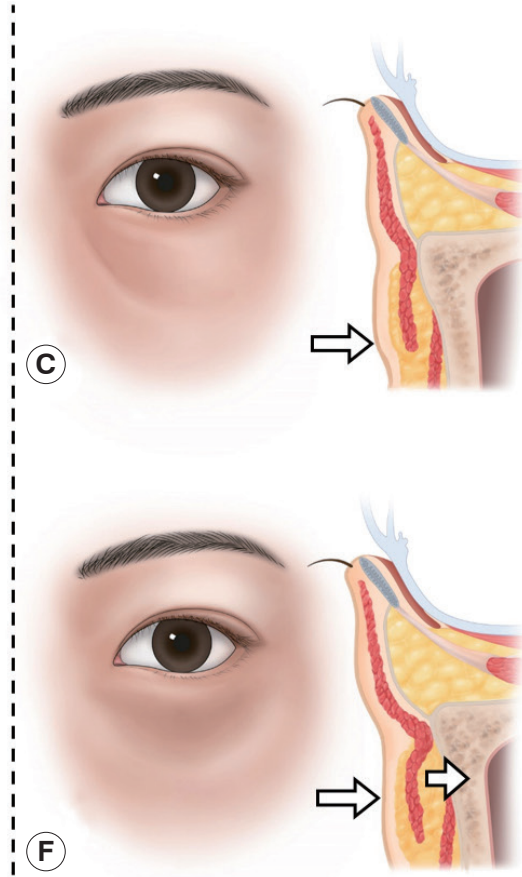


prominent difference in the thickness of the skin and soft tissue between the orbital part and the cheek part, we think that the groove seems more depressed. Whether or not there is a true osteocutaneous ligament, when ligamentous structures or fibrotic attachments are involved at the orbital rim junction, we can see finer and more prominent indentation than when only atrophy of the skin and soft tissue is present. (2) When contracting the orbital part of the OOM or squinting, the thickness of the OOM increases, leading to puckering of the periorbital skin, and the appearance of the tear trough deformity then becomes more severe (Fig. 3D). (3) When intraorbital fat is herniated, the appearance of a tear trough deformity can be aggravated (Fig. 3E). Usually, the palpebral portion of the lower eyelid skin becomes thinner and depressed [1]. However, some people show a swollen palpebral portion of skin due to bulging of the intraorbital fat [19]. Even though a prominent prolapse of intraorbital fat would require surgical correction, such as removal or redistribution of that fat and a lower blepharoplasty, we can improve GIR caused by mild intraorbital fat herniation using filler injections. (4) When soft tissue atrophies inferior to the arcus marginalis, causing depression of the anteromedial region and mid-cheek, which occurs upon the resorption of the malar bone, the HIR manifests as a groove [20]. In such cases, the volume deficiency of the broad flat area with a groove requires

\section{Fig. 4. The vessels of the infraorbital region}

Important vessels of the face of which practitioners should be cautious when treating grooves or hollowness in the infraorbital region. $\mathrm{Aa}$, angular artery; $\mathrm{Av}$, angular vein; 00 , orbicularis oculi muscle; ZFa, zygomaticofacial artery; IOa, infraorbital artery; ITFa, infraorbital trunk of facial artery; Fv, facial vein; Fa, facial artery.

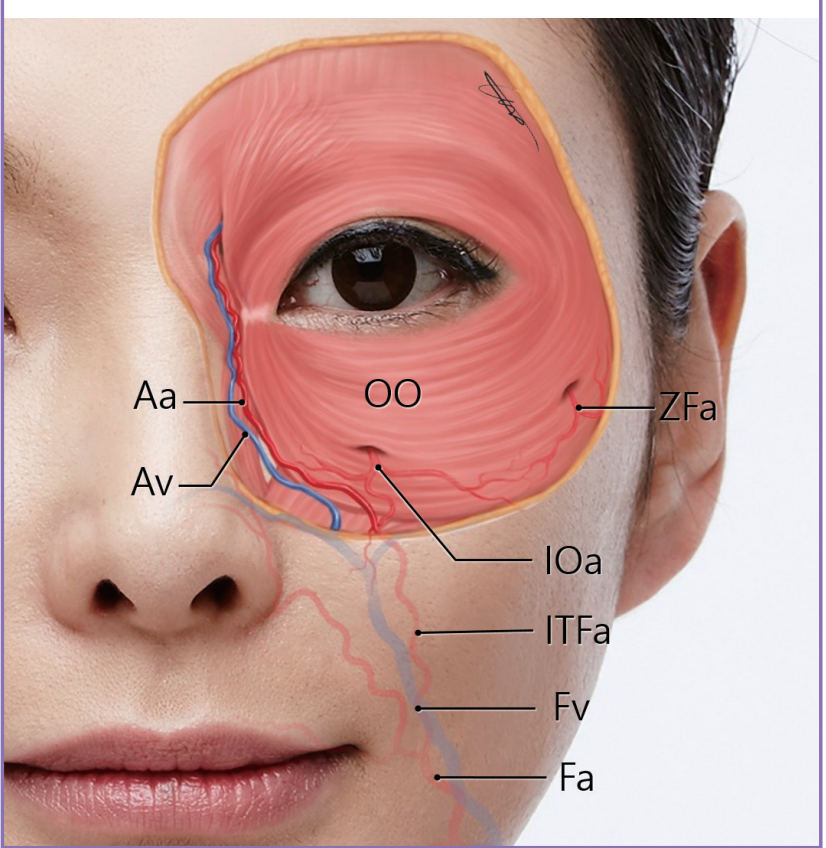

compensation (Fig. 3F).

\section{TREATMENT USING SOFT-TISSUE FILLER}

Before treatment, the pathways of the main vessels and nerves should be checked to ensure that the filler injection is safe. There are many important vessels and nerves in the infraorbital region, such as the infraorbital artery and nerve, angular artery and facial vein, a detoured branch of the facial artery (infraorbital trunk of the duplex facial artery), and the zygomaticofacial artery and nerve (Fig. 4). The infraorbital artery and nerve arise from the infraorbital foramen on the vertical line of the medial pupil at about $0.6-1.0 \mathrm{~cm}$ below the orbital rim [21]. The zygomaticofacial artery and nerve arise from the foramen on the vertical line of the outer margin of the lateral orbital rim at about $0.5-1.0 \mathrm{~cm}$ below the horizontal line of the lateral canthus and pass along the inferior border of the suborbicularis oculi fat (SOOF) [22].

If we want to use a needle, an injection along the orbital rim margin is relatively safe in terms of avoiding injury to the main vessels. The filler should be injected all around the orbital rim under the dermis and muscle using linear threading and the droplet technique (Fig. 5A). However, caution should be taken

\section{Fig. 5. Injection technique and points for the treatment}

(A) Injection around the orbital rim by linear threading, using the droplet technique. (B) Entry point for an injection to treat a groove when using a cannula. (C) Entry point for an injection to treat hollowness when using a cannula. IOF, infraorbital foramen.

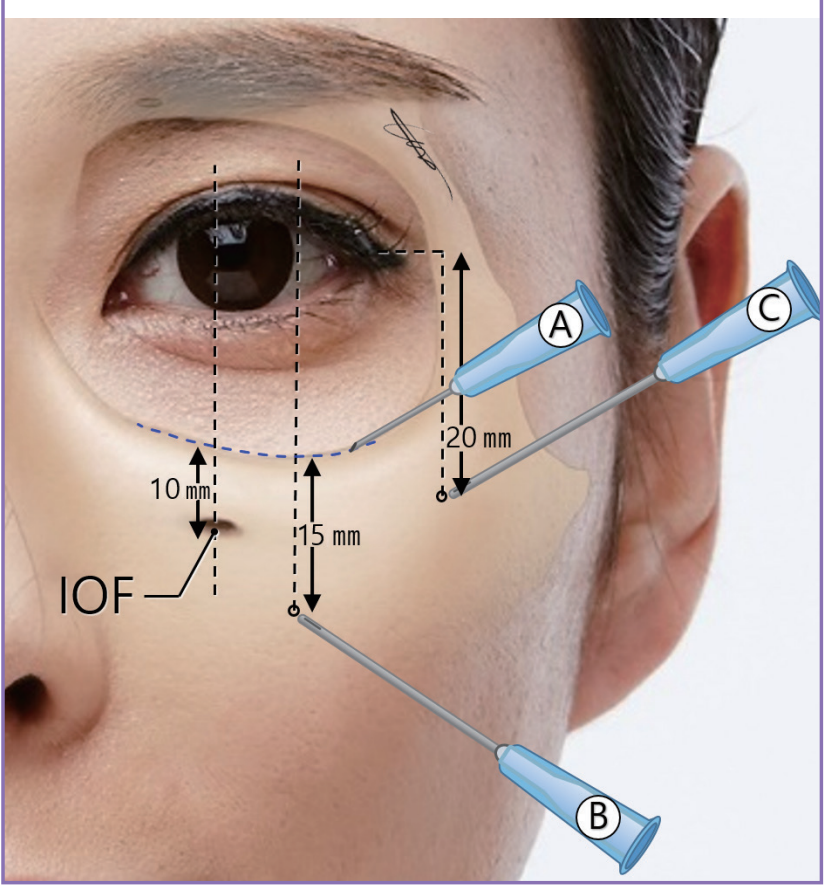


to avoid injuring the main vessels in the lower portion. The position of the facial artery and vein and the medial branch of the infraorbital artery under the nasojugal groove can be predicted $[16,17]$. Then, when injecting into the nasojugal groove area with a needle, nothing should be injected too deeply in order to avoid the possibility of severe bleeding and hematoma.

According to the above-discussed causes of GIR and HIR, an optimal type of filler and treatment method should be chosen. A pure hyaluronic acid (HA) filler is safe and effective for the infraorbital region because beading and irregularity are very common in the thin skin and subcutaneous tissue around this region, and the HA filler can be removed by the enzyme hyaluronidase [23]. Regarding the consistency of the filler, very soft filler is needed for subdermal injections under the thin skin of the infraorbital region to ensure a smooth surface without bulging or visible irregularities. The consistency of the HA filler can be controlled via dilution with lidocaine and saline. According to the latest study examining the dilution effect of pure HA filler via electron microscopy, there was no change in the ultrastructure after the dilution of 3 commonly used HA fillers [24]. Next, when injecting the filler, a cannula or needle can be chosen depending on various conditions; a needle is better for making fine contours for injection into a subdermal area above the OOM in a relatively safe plane, but a cannula is recommended to avoid vascular compromise when injecting filler into a deep fat layer under the OOM to compensate for the volume and to release the fibrotic ligamentous structure [25]. Using a cannula reduces the potential for serious bleeding and vascular compromise in the deep plane of the infraorbital region [1].

When there is only a mild indentation around the orbital rim and nasojugal region, a soft filler can be injected into the subcutaneous fat tissue. If the fat layer is insufficient for this, we can inject filler into the orbicularis muscle layer. After filling the main depression of a groove, this requires the injection of more diluted HA filler into the superficial subdermal space to even out the skin surface without irregularities. Because the skin and subcutaneous fat layer are thin in the periorbital area, the surface should be smoothed by the expansion of skin using a tenting technique [25]. When there is a relatively deep and fine indentation, whether or not it is truly a osteocutaneous ligament, the tethering effect of this ligamentous structure can be reduced by undermining and releasing fibrotic dense connective tissue and condensing the muscular attachment using a cannula prior to the filler injection. We can check whether there are dense ligamentous structures to make a fine indentation via the pushing test. When there is a fibrotic adhesion of the skin to the bone, the indentation line does not move, even when the skin and soft tissue are pushed forward. Based on photos of the cross-section- al view of injected fat into the infraorbital area on computed tomography in a recent study, injected fat pushes the depressed portion upward after the release of fibrotic ligamentous connective tissue around the arcus marginalis [26].

The safe entry point for release and injection using a cannula is $1.5-2.0 \mathrm{~cm}$ distant from the orbital rim on the vertical midline of the lateral limbus (Fig. 5B) [21,22]. After the release of dense fibrotic adhesions, a soft filler is injected into this released intermuscular and submuscular space. After filling the main groove, injecting diluted HA filler into the subdermal superficial space is required, as in cases of mild indentation, to improve the fine depressed line and to even out the surface around the orbital rim.

When there is mild prolapse of the intraorbital septal fat or broad flattening of the infraorbital region with or without a fine indentation, we can improve the GIR and HIR by restoring the volume deficiency using filler. If there is a severe indentation around the orbital rim, we first need to release the fibrotic attachment, and then perform the next step. The ideal injection entry point of a cannula for volume deficiency of the infraorbital region is $2 \mathrm{~cm}$ below the lateral canthus horizontal line, on the vertical line of the lateral orbital rim inner margin (Fig. 5C). Considering the injection plane, we usually perform a deep plane injection first and then a subdermal injection to even out the surface, as discussed above. We should inject the filler slightly under the infraorbital groove and hollowness, including the deep fat, SOOF in the middle, and the lateral orbit to push up the depressed portion from below [10]. However, the SOOF is not located in the medial part of the orbital area based on the vertical line of the medial limbus [25], so the filler should be injected under the muscle near the periosteum on the medial orbit. Because the submuscular space of the medial orbit is attached to the periosteum very densely, it is difficult to inject the filler into the space between the muscles and bone. After filling the volume, the procedure is completed by the superficial injection of diluted filler to remove the residual finely depressed line around the orbital rim and to smooth the surface.

In particular, a superficial injection is necessary for fine depressions at the medial end; the area near the medial canthus is very thin due to the deficiency of subcutaneous tissue volume, so there is insufficient room for a deep injection [1].

\section{CONCLUSIONS}

The safe and optimal treatment of GIR or HIR requires effective filler treatment methods based on the definitions and classification presented in this study. GIR can be differentiated into nasojugal groove (or fold), tear trough deformity, and palpebromalar groove; these conditions are caused by the herniation of 
intraorbital fat, atrophy of the skin and subcutaneous fat, contraction of the orbital part of OOM or squinting, and malar bone resorption.

Different treatment procedures should be chosen for GIR and HIR depending on its cause and anatomical characteristics. Appropriately choosing between a needle and a cannula and selecting the optimal type of filler and injection method facilitate the performance of a safe and successful procedure.

\section{NOTES}

\section{Conflict of interest}

No potential conflict of interest relevant to this article was reported.

\section{Ethical approval}

The study was performed in accordance with the principles of the Declaration of Helsinki. Written informed consents were obtained.

\section{Patient consent}

The patients provided written informed consent for the publication and the use of their images.

\section{REFERENCES}

1. Hirmand H. Anatomy and nonsurgical correction of the tear trough deformity. Plast Reconstr Surg 2010;125:699-708.

2. Haddock NT, Saadeh PB, Boutros S, et al. The tear trough and lid/cheek junction: anatomy and implications for surgical correction. Plast Reconstr Surg 2009;123:1332-40.

3. Costa CR, Kordestani R, Small KH, et al. Advances and refinement in hyaluronic acid facial fillers. Plast Reconstr Surg 2016;138:233e-236e.

4. Whitnall SE. The anatomy of the human orbit and accessory organs of vision. 2nd ed. London: Oxford University Press; 1932.

5. Loeb R. Naso-jugal groove leveling with fat tissue. Clin Plast Surg 1993;20:393-400.

6. Flowers RS. The tear trough deformity and its correction. Presented at the Annual Meeting of the California Society of Plastic Surgeons; 1970; Monterey, USA.

7. Hwang K. Eponym of naso-jugal fold and tear trough.J Craniofac Surg 2016;27:1350-3.

8. Yang C, Zhang P, Xing X. Tear trough and palpebromalar groove in young versus elderly adults: a sectional anatomy study. Plast Reconstr Surg 2013;132:796-808.

9. Hwang K, Kim HJ, Kim H, et al. Origin of the lower orbicu- laris oculi muscle in relation to the nasojugal groove. J Craniofac Surg 2015;26:1389-93.

10. Wong CH, Hsieh MK, Mendelson B. The tear trough ligament: anatomical basis for the tear trough deformity. Plast Reconstr Surg 2012;129:1392-402.

11. Muzaffar AR, Mendelson BC, Adams WP Jr. Surgical anatomy of the ligamentous attachments of the lower lid and lateral canthus. Plast Reconstr Surg 2002;110:873-84.

12. Flowers RS. Periorbital aesthetic surgery for men: eyelids and related structures. Clin Plast Surg 1991;18:689-729.

13. Mendelson BC, Muzaffar AR, Adams WP Jr. Surgical anatomy of the midcheek and malar mounds. Plast Reconstr Surg 2002;110:885-96.

14. Stutman RL, Codner MA. Tear trough deformity: review of anatomy and treatment options. Aesthet Surg J 2012;32: 426-40.

15. Kim HJ, Seo KK, Lee HK, et al. Botulinum filler clinical anatomy. Seoul: Hanmi Medical Publishing Co.; 2015.

16. Yang HM, Lee JG, Hu KS, et al. New anatomical insights on the course and branching patterns of the facial artery: clinical implications of injectable treatments to the nasolabial fold and nasojugal groove. Plast Reconstr Surg 2014;133: 1077-82.

17. Koh KS, Kim HJ, Oh CS, et al. Branching patterns and symmetry of the course of the facial artery in Koreans. Int J Oral Maxillofac Surg 2003;32:414-8.

18. Bosniak S, Cantisano-Zilkha M, Purewal BK, et al. Defining the tear trough. Ophthal Plast Reconstr Surg 2007;23:2545.

19. Lambros V. Observations on periorbital and midface aging. Plast Reconstr Surg 2007;120:1367-76.

20. Mendelson BC, Hartley W, Scott M, et al. Age-related changes of the orbit and midcheek and the implications for facial rejuvenation. Aesthetic Plast Surg 2007;31:419-23.

21. Scheuer JF 3rd, Sieber DA, Pezeshk RA, et al. Anatomy of the facial danger zones: maximizing safety during soft-tissue filler injections. Plast Reconstr Surg 2017;139:50e-58e.

22. Pessa JE, Rohrich RJ. Clinical anatomy of the face. Kim IH, Yoon ES, Translators. Seoul: Jeong woo Medical Book; 2012.

23. Carruthers J, Carruthers A, Humphrey S. Introduction to Fillers. Plast Reconstr Surg 2015;136(5 Suppl):120S-131S.

24. Flynn TC, Thompson DH, Hyun SH, et al. Ultrastructural analysis of 3 hyaluronic acid soft-tissue fillers using scanning electron microscopy. Dermatol Surg 2015;41 Suppl 1:S14352.

25. Cotofana S, Schenck TL, Trevidic P, et al. Midface: clinical anatomy and regional approaches with injectable fillers. 
Plast Reconstr Surg 2015;136(5 Suppl):219S-234S.

26. Stern CS, Schreiber JE, Surek CC, et al. Three-dimensional topographic surface changes in response to compartmental volumization of the medial cheek: defining a malar augmentation zone. Plast Reconstr Surg 2016;137:1401-8. 